\title{
A 12-Year Retrospective Study of the Prevalence of Anticholinergic Polypharmacy and Associated Outcomes Among Medicare Patients with Overactive Bladder in the USA
}

\author{
Noll L. Campbell ${ }^{1,2} \cdot$ Lisa Hines $^{3} \cdot$ Andrew J. Epstein $^{4} \cdot$ David Walker $^{5} \cdot$ Amy Lockefeer $^{5} \cdot$ Aki Shiozawa $^{5}(0$
}

Accepted: 19 September 2021 / Published online: 8 November 2021

(c) The Author(s) 2021

\begin{abstract}
Background and Objective Antimuscarinics, drugs with anticholinergic properties, are frequently prescribed for overactive bladder, and anticholinergic burden is associated with adverse events. The "Polypharmacy: Use of Multiple Anticholinergic Medications in Older Adults" (Poly-ACH) measure was developed by the Pharmacy Quality Alliance and is used by the Centers for Medicare and Medicaid Services. Using the Poly-ACH measure, we assessed the prevalence of anticholinergic polypharmacy among Medicare patients in the USA with overactive bladder and determined associations between polypharmacy and medical conditions, care, and spending.

Methods This was a retrospective cohort study of Medicare beneficiaries with overactive bladder (coverage period: 20062017). Anticholinergic polypharmacy, measured by the Poly-ACH, was defined as concurrent use of two or more anticholinergics, each with two or more prescription claims on different dates of service for $\geq 30$ cumulative days. Change in annual frequency of anticholinergic polypharmacy was assessed using logistic regression. Associations between anticholinergic polypharmacy over 3 years and falls, fractures, mental status, and medical care spending were assessed with longitudinal regression models.

Results In total, 226,712 patients contributed 940,201 person-years of follow-up after overactive bladder diagnosis. The share of patients meeting the Poly-ACH definition was 3.3\% in 2006 and $1.7 \%$ in 2017. Women and nursing home residents had higher risks of anticholinergic polypharmacy. Having 1 year or more of positive Poly-ACH status in the 3 years prior was associated with higher rates of all outcomes.

Conclusions Anticholinergic polypharmacy was uncommon among older adults with overactive bladder. Prevalence was higher among women and nursing home residents, and it was associated with negative outcomes, highlighting potential longitudinal implications of anticholinergic burden.
\end{abstract}

Aki Shiozawa

aki.shiozawa@astellas.com

1 Purdue University College of Pharmacy, West Lafayette, IN, USA

2 Indiana University Center for Aging Research, Indianapolis, IN, USA

3 Pharmacy Quality Alliance, Alexandria, VA, USA

4 Medicus Economics, Milton, MA, USA

5 Medical Affairs, U.S., Astellas Pharma Global Development, Inc., 1 Astellas Way, Northbrook, IL 60062, USA

\section{Key Points}

Anticholinergic polypharmacy, as defined by the "Polypharmacy: Use of Multiple Anticholinergic Medications in Older Adults" measure, was uncommon among Medicare beneficiaries with overactive bladder. Prevalence was higher among women and long-term nursing home residents.

Positive anticholinergic polypharmacy status was significantly associated with a greater number of negative outcomes, including falls, fractures, and altered mental status, as well as higher medical spending compared with negative status, drawing attention to the longitudinal implications of anticholinergic burden. 


\section{Introduction}

Overactive bladder (OAB) comprises a constellation of urinary symptoms typically characterized by urgency, with or without urge urinary incontinence, and accompanied by frequency and nocturia $[1,2]$. A review of the epidemiological literature indicates that the estimated overall prevalence of $\mathrm{OAB}$ in the USA is between 16.5 and $23.3 \%$, with women and minorities accounting for the majority of cases [3]. Currently, the total costs associated with $\mathrm{OAB}$ management in the USA, including all medical (direct and indirect) and indirect non-medical costs, are US $\$ 82.6$ billion [4].

Antimuscarinic agents are the current mainstay of $\mathrm{OAB}$ pharmacotherapy [5]. Among older adults (those aged 65 years and older), previous research using Medicare claims has shown that $75 \%$ of Medicare patients with OAB use at least one antimuscarinic drug [6]. However, antimuscarinic agents are part of the class of oral anticholinergic medications, which are associated with numerous central and peripheral adverse events [7-9]. Among older adults, anticholinergic use has been linked to impairments in physical performance, the reduced ability to perform activities of daily living, and an increased risk of dementia [10-12]; cumulative exposure to anticholinergic medications increases the risk of these events [10-12]. As a result, the most recent version of the American Geriatrics Society Beers Criteria includes the recommendation to avoid drugs with strong anticholinergic properties [13]. Notably, all antimuscarinics approved for the treatment of OAB in the USA are considered strong anticholinergics and have been listed as medications to avoid in certain circumstances [13].

Currently, the prevalence and economic implications of anticholinergic burden are largely derived from studies conducted among community-dwelling populations and include individuals across a broad age range. One study found that among commercially insured patients with OAB in the USA, approximately $65 \%$ had some level of anticholinergic burden. Furthermore, healthcare resource utilization and costs directly increased with a higher level of anticholinergic burden [14]. Likewise, a study of 3344 community-dwelling older adults found that increasing levels of anticholinergic burden were also associated with higher costs [15]. However, given the lack of studies conducted specifically among older individuals with $\mathrm{OAB}$, the prevalence and economic consequences of anticholinergic burden among this population are not understood.

The Pharmacy Quality Alliance developed a health plan performance measure, titled "Polypharmacy: Use of Multiple Anticholinergic Medications in Older Adults" (Poly-ACH). Poly-ACH is a medication safety measure to identify co-prescribing of strong anticholinergic medications in older adults because of an associated risk of cognitive decline [15]. The measure defines anticholinergic polypharmacy as the use of at least two anticholinergic medications that overlap for at least 30 days in the measurement year [16]. While there are several scales to measure anticholinergic burden, Poly-ACH is especially important because of its policy relevance. Recently, the Centers for Medicare and Medicaid Services has adopted it for use in its Medicare Part D plan reporting efforts (Patient Safety Reports). The Centers for Medicare and Medicaid Services intends to include health-plan-specific Poly-ACH scores in its publicly available Display Pages in 2021 (using 2019 data) and is evaluating whether to include the Poly-ACH measure as part of their publicly available health-plan-specific Star Ratings in the future [17]. However, anticholinergic burden, as defined by the Poly-ACH measure, has not yet been widely studied.

Therefore, there is a need to understand the extent of anticholinergic burden and the associated clinical and economic outcomes among older adults with OAB, particularly across a broader range of residential settings that includes both community-dwelling and institutionalized individuals. Our study had two objectives. We first sought to characterize secular trends in the annual frequency of anticholinergic polypharmacy as measured by the Poly$\mathrm{ACH}$, overall and by important beneficiary attributes. We also assessed the associations between positive Poly-ACH status and clinical and economic outcomes, including the risk of falls, fractures, altered mental status (AMS; chosen as AMS/delirium is a shorter term outcome and appropriate to the timeframe of this work $[11,18])$, and medical care spending.

\section{Methods}

\subsection{Study Design and Data}

This was a retrospective cohort study using Medicare enrollment and claims data for individuals covered under traditional Medicare from 1 January, 2006 through 31 December, 2017. Enrollment files contain monthly information on individuals' enrollment in each part of Medicare, including Part D (pharmacy) coverage, in addition to demographic information, residential location (at the 5-digit ZIP code level), and date of death (from Medicare administrative files). Claims data are available for all medical services and outpatient prescription drugs covered by the program and are organized into data files based on the nature and source of the claim. MedPAR and outpatient files include institutional claims from hospitals for inpatient and outpatient services and from nursing homes for short-stay "skilled" admissions. The Carrier file includes fee-for-service claims submitted by professional providers, 
including physicians, physician assistants, clinical social workers, and nurse practitioners. The Part D files include all information on all transactions covered by a beneficiary's Medicare prescription drug plan. This study was reviewed and approved by the New England Institutional Review Board (\#1-6735-1).

\subsection{Core Study Sample}

The core study sample included beneficiaries with OAB who were US residents, aged $\geq 65$ years, and had continuous enrollment in Medicare Parts A, B, and D and no enrollment in Medicare Part C (Medicare Advantage). The presence of $\mathrm{OAB}$ was determined by one or more inpatient or outpatient facility claims or at least two Carrier file claims on separate dates with an OAB diagnosis code (Table 1 of the Electronic Supplementary Material [ESM] $[19,20]$. Enrollment and medical claims data from 2005 were used to assess an individual's earliest medical claim with an OAB diagnosis code for those who enrolled in Medicare prior to the start of Part D (1 January, 2006). Individuals were excluded if they had a diagnosis code for any of the following conditions on at least one medical claim at any time during the study period: neurogenic bladder/neurogenic detrusor overactivity, pregnancy, malignant neoplasm, renal impairment, hepatic insufficiency, trauma, or organ transplantation, as these conditions have similar symptomology to $\mathrm{OAB}$ but unique pathology that may incorrectly influence associations being evaluated. A procedure code for any of the following was also exclusionary: non-indwelling bladder catheter, sterile intermittent catheter kit, indwelling bladder catheter, or transplant. For both study objectives, the unit of analysis was the person-year; thus, incomplete calendar years (i.e., when a patient entered after 1 January or exited before 31 December) were excluded.

\subsection{Anticholinergic Polypharmacy as Measured by the Poly-ACH}

The National Drug Code lists required to calculate the Poly-ACH measure as specified were obtained from the Pharmacy Quality Alliance. The anticholinergic medications targeted for the measure are Drugs with Strong Anticholinergic Properties from the 2019 American Geriatrics Society Beers Criteria [13]. A binary indicator was used to indicate whether beneficiaries had Poly-ACH in a given calendar year (yes/no; positive/negative) based on the Pharmacy Quality Alliance definition. This definition included the following four components:
1. An individual had positive evidence of Poly-ACH when the individual had concurrent use for $\geq 30$ cumulative days of two or more unique anticholinergic medications, each with two or more prescription claims on different dates of service during the measurement year.

2. Concurrent use was identified using the dates of an individual's prescription claims and corresponding days' supply. The days of concurrent use were calculated as the count of days during the measurement year with overlapping days' supply for two or more unique anticholinergic medications. Days' supply or overlap that occurred after the end of the measurement year were excluded.

3. If multiple prescription claims for the same anticholinergic medication (active ingredient) were filled on the same day, the number of days covered by the anticholinergic medication were calculated using the prescriptions with the longest days' supply.

4. If multiple prescription claims of the same anticholinergic medication (active ingredient) were filled on different days with overlapping days' supply, each day in the measurement year was counted only once toward the Poly-ACH status determination. There was no adjustment for early fills or overlapping days' supply.

It should be noted that the Poly-ACH measure denominator excludes individuals in hospice care (during the measurement year) though this exclusion was not applied for the study.

\subsection{Statistical Analysis: Trends in Poly-ACH}

To characterize secular trends in the annual frequency of positive Poly-ACH status overall and by important beneficiary attributes, the following covariates were considered: age category (65-74, 75-84, 85+ years), female sex, Census region (Northeast, South, Midwest, West), and nursing home use category (none, short term, or long term). Regarding the classification of nursing home use, individuals were characterized according to their highest level of nursing home use during each calendar year (e.g., individuals who switched from a short-term to long-term status were classified as long term).

Counts and percentages were calculated for all covariates stratified by Poly-ACH status (yes/no) and compared with $\chi^{2}$ tests. Logistic regression was used to model Poly-ACH as predicted by a vector of indicator variables for the calendar year. Stratified trends were calculated for each stratification variable one at a time.

Adjusted overall trends were calculated by controlling for the stratification variables (in addition to calendar year) as main effects in the logistic regression. Predictive margins 
on the probability scale and their $95 \%$ confidence intervals (CIs) were calculated from each logistic regression model. Estimated standard errors were adjusted to be robust to heteroskedasticity of unknown form and for multiple yearly observations per person.

\subsection{Statistical Analysis: Associations between Poly-ACH Status and Outcomes}

The association between lagged Poly-ACH and current outcomes were estimated using longitudinal regression analyses; this approach, also known as panel regression modeling, has previously been applied to similar efforts [21]. As shown in Fig. 1, for each year " $t$ " during which an outcome was measured, the exposure was the count over the prior 3 years $(t-1, t-2$, and $t-3)$ that a beneficiary experienced Poly-ACH (i.e., between zero and three). Control variables were measured during year $t-1$. As a result of this design, the sample was limited to individuals who met the additional criterion of having at least 4 consecutive years of enrollment ( 3 years of baseline/lagged Poly-ACH and 1 year of follow-up). Thus, the subset of individuals within the core sample who met this additional criterion formed the analytic sample for the second study objective. Beneficiaries contributed observations to the analytic dataset in a rolling manner; for example, a beneficiary with 6 consecutive years of data contributed three observations, starting with their fourth year.

Clinical and economic outcomes assessed for this analysis included fall, fracture, AMS, and total medical spending. The first three were specified as binary variables (yes/no) indicating whether an individual had a medical claim with a relevant diagnosis (International Classification of Diseases, 9th Revision, Clinical Modification and International
Classification of Diseases, 10th Revision, Clinical Modification or service code [Current Procedural Terminology Code]) for a given outcome during each calendar year (Table 2 of the ESM). Total medical spending was calculated as the sum of total dollars paid by Medicare, other payers, and patients on yearly medical care as found on inpatient, skilled nursing facility, outpatient, and Carrier claim files and was inflated to 2017 US dollars using the Gross Domestic Product Price Index [22]. Patient-year-level observations were compared based on their distribution of lagged 3 years with Poly-ACH two ways: any years with Poly-ACH during the prior 3 years vs no Poly-ACH ( $1=$ any, $0=$ none $)$, and number of years with Poly-ACH (1, 2 or 3$)$ vs no Poly-ACH (0).

Regression modeling was used to assess the independent statistical associations between anticholinergic polypharmacy exposures and each outcome. Models were specified as logistic regression for binary outcomes and generalized linear models using a $\log$ link and a Poisson family distribution (as determined by the modified Park test) for total medical spending [23]. Controls included covariates (as categorical variables), specifically patient demographics and medical conditions, particularly those that were associated with anticholinergic use. These covariates were measured during the prior year and lagged outcome during the prior 3 years. Demographics were observed during year 1 and included age category, sex, census region, nursing home category, and calendar year. Medical conditions included those associated with a higher risk of falls and fractures from Szabo et al. [19] (e.g., Parkinson's disease, dementia, hypertension, and diabetes), Elixhauser comorbidities [14, 24], and conditions indicated for treatment with an anticholinergic medication (Fall/fracture-related diagnostic codes: Table 2 of the ESM; Conditions: Table 3 of the ESM).
Fig. 1 Longitudinal data structure: patients were observed for at least 4 consecutive years including three baseline/lagged Polypharmacy: Use of Multiple Anticholinergic Medications in Older Adults (measure) years and 1 follow-up year. *Beneficiaries contributed observations to the analytic dataset in a rolling manner; for example, a beneficiary with 6 consecutive years of data contributed three observations, starting with their fourth year

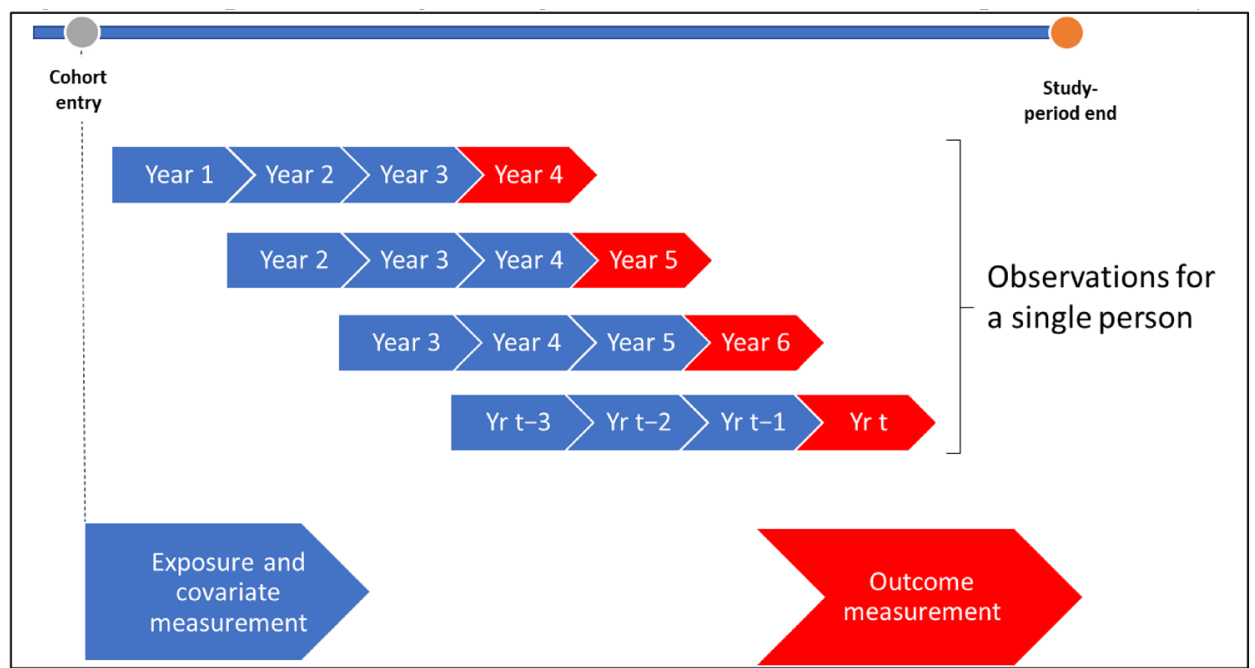

Abbreviations: $y r=$ year 
Predictive margins and average marginal effects on the original scale (i.e., probability, dollars) and their 95\% CIs as well as relative risks were calculated from each regression model. Estimated standard errors were adjusted to be robust to heteroskedasticity of unknown form and for multiple yearly observations per person.

\section{Results}

The core sample initially included 226,712 beneficiaries with $1,926,495$ calendar years of data. After including only complete person-years that occurred after the first observed OAB diagnosis, the final sample consisted of a total of 940,201 years of data from 226,712 beneficiaries. For the second study objective, the additional requirement of having at least 4 consecutive complete calendar years of data reduced the sample to 405,343 person-year observations from 105,608 beneficiaries.

Person-years with positive Poly-ACH tended to be from individuals who were older, more frequently female, more commonly from the Midwest and South Census regions, and more frequent users of long-term care facilities (Table 1). Regarding anticholinergic use over the entire study period (2006-2017), an average of $26 \%$ of all beneficiaries in each year had at least one claim for an anticholinergic medication, $3 \%$ had at least two claims for at least two anticholinergic medications, and 3\% met the criteria for Poly-ACH. Further details of anticholinergic use in the cohort are available in Table 4 of the ESM.

\subsection{Trends in the Annual Frequency of Positive Poly-ACH Status}

Overall, Poly-ACH was infrequent and declined over the study period (2006-2017) (Table 2). The percentage of beneficiaries with Poly-ACH was $3.3 \%$ in 2006, $1.5 \%$ in 2015, and $1.7 \%$ in 2017. Specific subgroups of the study population were consistently at a higher risk of anticholinergic polypharmacy, including women and users of nursing home care. Regression adjustment for age, sex, geographic region, and nursing home use had minimal effect on the anticholinergic polypharmacy estimates in the most recent years (Fig. 2).

\subsection{Clinical and Economic Outcomes Associated with Positive Poly-ACH Status}

Having at least 1 year with positive Poly-ACH status over the prior 3 years was associated with a 1.26 greater risk of any fall (absolute difference of 1.0 percentage points; $95 \%$ CI $0.07-1.30 ; p<0.001)$, a 1.14 greater risk of fracture (absolute difference of 0.4 percentage points; $95 \%$
CI $0.20-0.7 ; p=0.001)$, a 1.31 greater risk of AMS (absolute difference of 0.8 percentage points; 95\% CI $0.50-0.10 ; p<0.001$ ), and 1.13 times higher medical care spending (absolute difference of US $\$ 1116 ; 95 \% \mathrm{CI}$ US\$677-US\$1556; $p<0.001$ ) (Table 3). The effect of having any anticholinergic polypharmacy in the prior 3 years did not vary systematically by the count of lagged years with Poly-ACH. For the annual medical care spending outcome, relative to no years of Poly-ACH, having 1 year of positive Poly-ACH was associated with US\$1163 higher spending, 2 years with US\$1169 higher spending, and 3 years with US\$964 (Table 3).

\section{Discussion}

Overall, this study found that anticholinergic polypharmacy is not common among Medicare beneficiaries with OAB. Regarding trends among those with anticholinergic polypharmacy, it was more common among women and those in long-term care settings.

Notably, although anticholinergic polypharmacy was infrequent among the chosen study population, a relationship between positive Poly-ACH status and negative outcomes was nonetheless observed. Specifically, patients who experienced anticholinergic polypharmacy in at least one of the prior 3 years had statistically significant and clinically meaningful higher risks of fall, fracture, and AMS, and higher annual medical care spending. These findings highlight the longitudinal nature of the consequences of anticholinergic polypharmacy, the implications of which have to an extent been previously evaluated. Indeed, a number of recent studies have found that exposure to certain anticholinergic medications is associated with an increased risk of negative outcomes, including dementia and falls/fractures, the latter of which has specifically been evaluated among older adults with OAB [11, 18, 19, 25, 26]. Furthermore, even the lowest levels of exposure were associated with an increased risk. While the current study was not designed to determine causality, the findings nonetheless contribute evidence to an association between exposure to multiple anticholinergics and negative long-term consequences, which should be explored further. The insights regarding these associations derived from the Poly-ACH measure have important implications for decision makers and care providers and should be considered when evaluating interventions to reduce exposure. Finally, it is possible that the number of older adults with $\mathrm{OAB}$ and positive Poly-ACH status is larger than the current study suggests, given that the estimated proportions of individuals with $\mathrm{OAB}$ derived from other studies using Medicare claims data (9-14\%) [27] are less than those that have been reported for the general US population (16.5-23.3\%) [3]. 
Table 1 Descriptive statistics (person-years over the entire study period)

\begin{tabular}{|c|c|c|c|c|}
\hline Variable name & Overall & No Poly-ACH & Yes Poly-ACH & $p$ value \\
\hline Sample size (person-years) & 940,201 & 919,963 & 20,238 & \\
\hline \multicolumn{4}{|l|}{ Age category, years, $N(\%)$} & $<0.001$ \\
\hline $65-74$ & $505,045(54 \%)$ & $496,468(54 \%)$ & $8577(42 \%)$ & \\
\hline $75-84$ & $280,448(30 \%)$ & $273,923(30 \%)$ & $6525(32 \%)$ & \\
\hline $85+$ & $154,708(16 \%)$ & $149,572(16 \%)$ & $5136(25 \%)$ & \\
\hline \multicolumn{4}{|l|}{ Sex, $N(\%)$} & $<0.001$ \\
\hline Male & $276,271(29 \%)$ & $274,173(30 \%)$ & $2098(10 \%)$ & \\
\hline Female & $663,930(71 \%)$ & $645,790(70 \%)$ & $18,140(90 \%)$ & \\
\hline \multicolumn{4}{|l|}{ Census region, $N(\%)$} & $<0.001$ \\
\hline Midwest & $270,545(29 \%)$ & $264,133(29 \%)$ & $6412(32 \%)$ & \\
\hline Northeast & $176,798(19 \%)$ & $173,855(19 \%)$ & $2943(15 \%)$ & \\
\hline South & $335,715(36 \%)$ & $328,082(36 \%)$ & $7633(38 \%)$ & \\
\hline West & $157,143(17 \%)$ & $153,893(17 \%)$ & $3250(16 \%)$ & \\
\hline \multicolumn{4}{|c|}{ Annual nursing home use category, $N(\%)$} & $<0.001$ \\
\hline No NH & $852,436(91 \%)$ & $837,354(91 \%)$ & $15,082(75 \%)$ & \\
\hline SNF only & $16,148(1.7 \%)$ & $15,407(1.7 \%)$ & $741(3.7 \%)$ & \\
\hline Long-term NH & $71,617(7.6 \%)$ & $67,202(7.3 \%)$ & $4415(22 \%)$ & \\
\hline \multicolumn{4}{|c|}{ Person-years by calendar year, $N(\%)$} & $<0.001$ \\
\hline 2007 & $40,092(4.3 \%)$ & $37,821(4.1 \%)$ & $2271(11 \%)$ & \\
\hline 2008 & $47,490(5.1 \%)$ & $45,321(4.9 \%)$ & $2169(11 \%)$ & \\
\hline 2009 & $53,039(5.6 \%)$ & $51,120(5.6 \%)$ & $1919(9.5 \%)$ & \\
\hline 2010 & $58,496(6.2 \%)$ & $56,706(6.2 \%)$ & $1790(8.8 \%)$ & \\
\hline 2011 & $63,877(6.8 \%)$ & $62,221(6.8 \%)$ & $1656(8.2 \%)$ & \\
\hline 2012 & $70,872(7.5 \%)$ & $69,426(7.5 \%)$ & $1446(7.1 \%)$ & \\
\hline 2013 & $80,647(8.6 \%)$ & $79,161(8.6 \%)$ & $1486(7.3 \%)$ & \\
\hline 2014 & $94,476(10 \%)$ & $93,026(10 \%)$ & $1450(7.2 \%)$ & \\
\hline 2015 & $112,614(12 \%)$ & $110,976(12 \%)$ & $1638(8.1 \%)$ & \\
\hline 2016 & $139,997(15 \%)$ & $138,125(15 \%)$ & $1872(9.2 \%)$ & \\
\hline 2017 & $178,601(19 \%)$ & $176,060(19 \%)$ & $2541(13 \%)$ & \\
\hline Sample size & 940,201 & 919,963 & 20,238 & \\
\hline
\end{tabular}

Medicare enrollment and claims data, calendar year 2006-2017

NH nursing home, Poly-ACH Polypharmacy: Use of Multiple Anticholinergic Medications in Older Adults (measure), $S N F$ skilled nursing facility

Although differences exist between study populations, the findings from this study are in line with those of previous studies. In the present study, having at least one year with positive Poly-ACH status was associated with higher risks of fall and fracture. Szabo et al. found that cumulative anticholinergic burden was also associated with an increased risk of falls and fractures among patients with $\mathrm{OAB}$ in a community setting; those with low, medium, and high levels of burden had a 23\%, 30\%, and 38\% higher risk, respectively, than those with no burden [19]. Additionally, Lozano-Ortega et al. found that healthcare resource utilization among outpatients with OAB increased with the level of anticholinergic burden. Furthermore, costs associated with falls and fractures also rose as the level of anticholinergic burden increased [14].

Limitations of this analysis included those that are inherent to administrative claims data. Ascertainment of conditions and treatments relied on administrative codes, which are subject to coding error. Furthermore, anticholinergic medications obtained over the counter would not be recorded; thus, the level of exposure observed in this study may be underestimated. Cases of OAB, along with outcomes such as fractures, falls, and AMS, may also be underreported. Furthermore, the denominator of the Poly-ACH measure is meant to exclude individuals in hospice care, but those individuals were not excluded from the current 
Table 2 Core counts of beneficiaries with OAB diagnosis with anticholinergic drug claims and Poly-ACH status by calendar year

\begin{tabular}{|c|c|c|c|c|c|}
\hline Calendar year & Total beneficiaries & $\begin{array}{l}1+\text { claims for } 1+\mathrm{ACH} \\
\text { drugs }\end{array}$ & $\begin{array}{l}1+\text { claims for } 2+\mathrm{ACH} \\
\text { drugs }\end{array}$ & $\begin{array}{l}2+\text { claims for } 2+\mathrm{ACH} \\
\text { drugs }\end{array}$ & Poly-ACH \\
\hline 2006 & 127,586 & $44,717(35 \%)$ & $13,763(11 \%)$ & $7244(6 \%)$ & $47504 \%)$ \\
\hline 2007 & 130,977 & $44,094(34 \%)$ & $13,068(10 \%)$ & $6564(5 \%)$ & $4359(3 \%)$ \\
\hline 2008 & 132,112 & $41,057(31 \%)$ & $11,001(8 \%)$ & $5461(4 \%)$ & $3654(3 \%)$ \\
\hline 2009 & 132,284 & $38,126(29 \%)$ & $9318(7 \%)$ & $4458(3 \%)$ & $3043(2 \%)$ \\
\hline 2010 & 134,079 & $36,933(28 \%)$ & $8917(7 \%)$ & $4082(3 \%)$ & $2692(2 \%)$ \\
\hline 2011 & 140,243 & $36,438(26 \%)$ & $8378(6 \%)$ & $3645(3 \%)$ & $2498(2 \%)$ \\
\hline 2012 & 150,959 & $35,520(24 \%)$ & $7095(5 \%)$ & $3125(2 \%)$ & $2167(1 \%)$ \\
\hline 2013 & 164,041 & $37,061(23 \%)$ & $7253(4 \%)$ & $3180(2 \%)$ & $2216(1 \%)$ \\
\hline 2014 & 179,873 & $38,468(21 \%)$ & $6779(4 \%)$ & $3014(2 \%)$ & $2167(1 \%)$ \\
\hline 2015 & 197,405 & $41,499(21 \%)$ & $7170(4 \%)$ & $3199(2 \%)$ & $2299(1 \%)$ \\
\hline 2016 & 214,415 & $45,680(21 \%)$ & $7808(4 \%)$ & $3471(2 \%)$ & $2500(1 \%)$ \\
\hline 2017 & 222,521 & $52,218(23 \%)$ & $9354(4 \%)$ & $4111(2 \%)$ & $3008(1 \%)$ \\
\hline
\end{tabular}

Source: Medicare enrollment and claims data, calendar year 2006-17

$A C H$ anticholinergic, $O A B$ overactive bladder, Poly-ACH Polypharmacy: Use of Multiple Anticholinergic Medications in Older Adults (measure)

study. However, because beneficiaries receiving hospice benefits represent less than $3 \%$ of all beneficiaries [28, 29], the impact of their inclusion is expected to be low. Additionally, because data on beneficiaries before their Medicare enrollment were unavailable, it is unknown to what extent any prior exposure to anticholinergic medications or other relevant variables may have affected the outcomes. Similarly, given the nature of the study design, there is the possibility of reverse causality; some of the study outcomes (especially falls and fractures) could have precipitated the prescribing of anticholinergic medications. In addition, the generalizability of study findings is limited to beneficiaries with $\mathrm{OAB}$ and continuous enrollment in traditional Medicare, including Part D.

Regarding the Poly-ACH measure, the binary indicator of exposure (yes/no) precludes the consideration of key aspects of cumulative anticholinergic drug use, including the number of days of overlap and total anticholinergic dose. Thus, it is not possible to ascertain the extent to which varying levels of exposure are associated with the current findings. To account for varying exposure times, future research using the Poly-ACH metric could examine cumulative increments (e.g., 30 days) in which Poly-ACH exists and the association with the study outcomes. Next, while positive Poly-ACH status occurred infrequently among the study population, there was nonetheless a correlation with the measured adverse outcomes. Given that the Poly-ACH measure does not assess characteristics such as medication potency or specific dose, additional research is needed to examine to what extent this relationship is driven by the level of anticholinergic exposure. Finally, this study focused on a sub-population of individuals (those with $\mathrm{OAB}$ ); the Poly-ACH measure was intended for the broader population of all Medicare beneficiaries. Thus, while the Poly-ACH measure may not be suitable for accountability purposes within specific subpopulations and regarding specific levels of anticholinergic exposure, it appears to have utility as a measure for safety monitoring or quality improvement purposes.

\section{Conclusions}

These findings contribute to the initial evidence base regarding the extent and implications of anticholinergic burden among older adults with OAB. Anticholinergic burden, as measured by the Poly-ACH metric, was relatively uncommon among Medicare beneficiaries. However, the relationship observed between anticholinergic polypharmacy and adverse outcomes supports the American Geriatrics Society Beers Criteria recommendation [13] that concurrent use of two or more anticholinergic medications should be avoided among older adults. 


\section{A Overall unadjusted results}

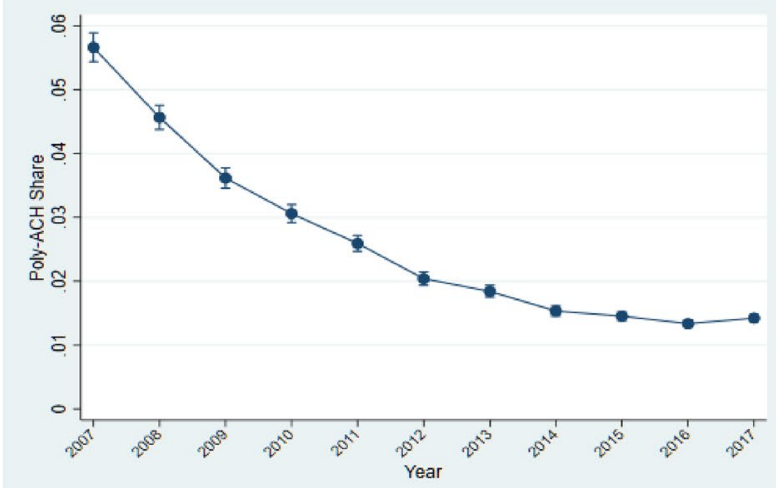

C Results stratified by age category

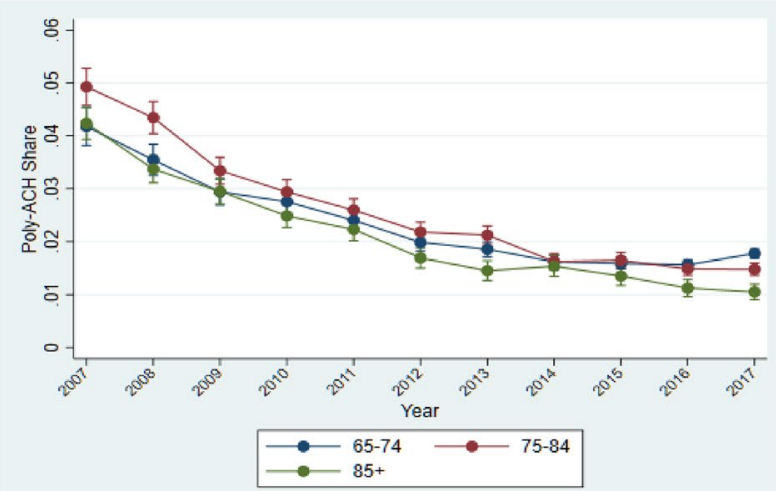

E Results stratified by Census Region

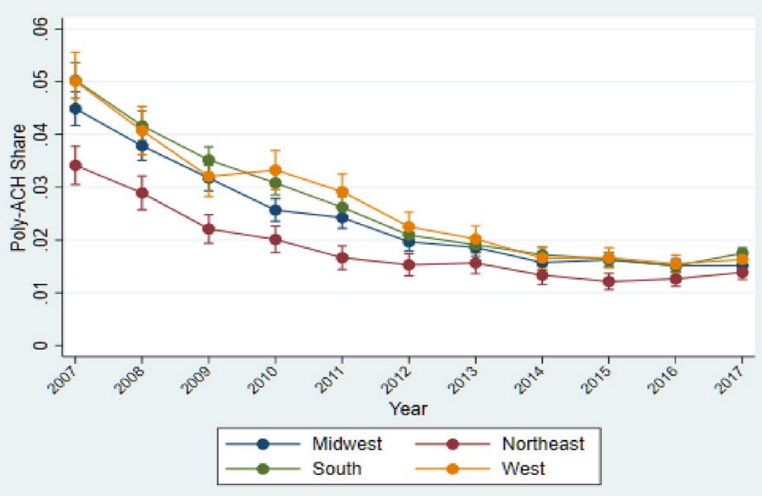

Fig. 2 Trends in Polypharmacy: Use of Multiple Anticholinergic Medications in Older Adults (measure) [Poly-ACH] status. Note: figure shows estimates of annual shares and their $95 \%$ confidence intervals. Source: Medicare enrollment and claims data, CY 2006-2017.

\section{B Overall adjusted results}

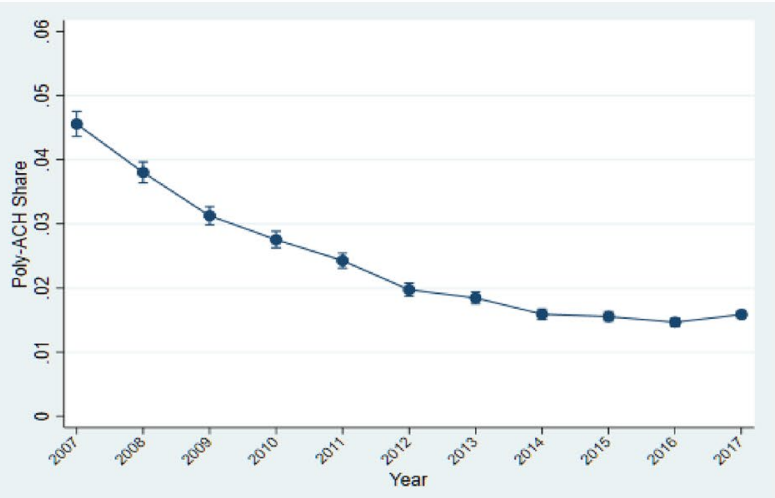

\section{Results stratified by sex}

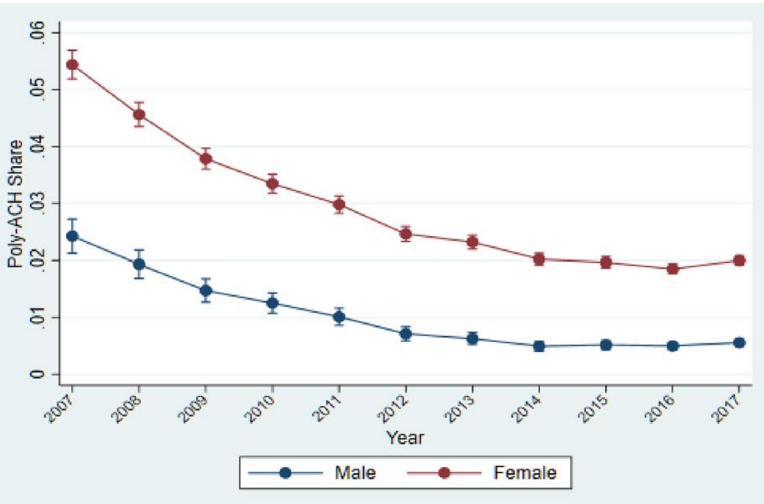

\section{F Results stratified by nursing home category}

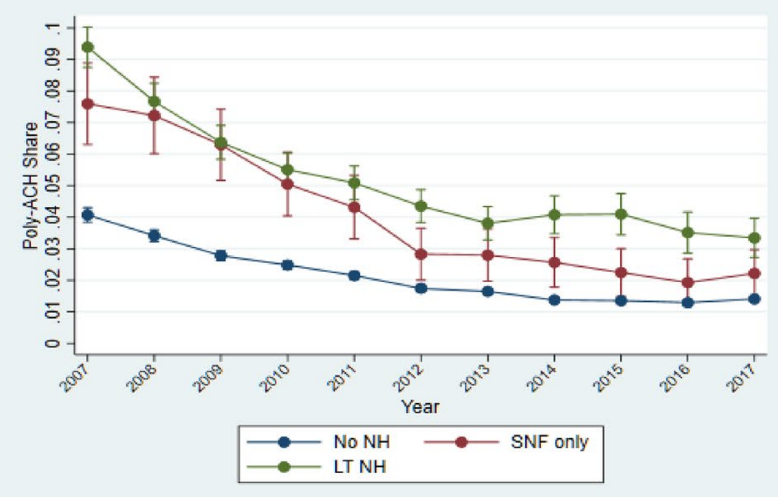

LT long-term, NH nursing home, Poly-ACH Polypharmacy: Use of Multiple Anticholinergic Medications in Older Adults (measure), $S N F$ skilled nursing facility 
Table 3 Adjusted clinical outcomes and spending for Medicare beneficiaries with overactive bladder by Poly-ACH status

\begin{tabular}{llllll}
\hline Outcome name & Comparison vs referent & $\begin{array}{l}\text { Mean risk } \\
\text { (referent) }\end{array}$ & $\begin{array}{l}\text { Mean risk } \\
(\text { comparison) }\end{array}$ & $\begin{array}{l}\text { Absolute risk } \\
\text { difference }(\%)\end{array}$ & $\begin{array}{l}p \text { value } \\
(\%)\end{array}$ \\
\hline
\end{tabular}

Annual indicator for any fall

Count of lagged Poly-ACH 1 yr vs 0 yrs 3.8

2 yrs vs 0 yrs $\quad 3.8$

3 yrs vs 0 yrs

Annual indicator for ER fall

Any lagged Poly-ACH $\quad 1+$ yrs vs 0 yrs 2.8

Count of lagged Poly-ACH 1 yr vs 0 yrs

2 yrs vs 0 yrs

3 yrs vs 0 yrs

Annual indicator for inpatient hospital fall

Any lagged Poly-ACH $1+$ yrs vs 0 yrs

Count of lagged Poly-ACH 1 yr vs 0 yrs

2 yrs vs 0 yrs

3 yrs vs 0 yrs

Annual indicator for any fracture

Any lagged Poly-ACH $1+$ yrs vs 0 yrs

Count of lagged Poly-ACH 1 yr vs 0 yrs

2 yrs vs 0 yrs

3 yrs vs 0 yrs

Annual indicator for ER fracture

Any lagged Poly-ACH $\quad 1+$ yrs vs 0 yrs

Count of lagged Poly-ACH 1 yr vs 0 yrs

2 yrs vs 0 yrs

3 yrs vs 0 yrs

Annual indicator for inpatient hospital fracture

Any lagged Poly-ACH $\quad 1+$ yrs vs 0 yrs

Count of lagged Poly-ACH 1 yr vs 0 yrs

2 yrs vs 0 yrs

3 yrs vs 0 yrs

Annual indicator for any AMS

Any lagged Poly-ACH $1+$ yrs vs 0 yrs

Count of lagged Poly-ACH 1 yr vs 0 yrs

2 yrs vs 0 yrs

3 yrs vs 0 yrs

Annual indicator for ER AMS

Any lagged Poly-ACH $\quad 1+$ yrs vs 0 yrs

Count of lagged Poly-ACH 1 yr vs 0 yrs

2 yrs vs 0 yrs

3 yrs vs 0 yrs

Annual indicator for inpatient hospital AMS

Count of lagged Poly-ACH 1 yr vs 0 yrs

2 yrs vs 0 yrs

3 yrs vs 0 yrs
4.9

4.6

4.9

3.6

3.7

3.4

3.7

0.7

1.0

1.1

1.0

1.0

0.7

3.7

3.7

3.7

3.7

1.4

1.4

1.4

1.4

1.1

1.1

1.1

1.1

2.6

2.6

2.6

2.6

1.5

1.5

1.5

1.5

4.2

4.2

3.9

4.2

1.7

1.8

1.7

1.7

1.4

1.4

1.2

1.3

3.4

3.2

3.5

3.6

1.9

1.8

1.9

2.0

$\begin{array}{ll}0.1 & 0.2\end{array}$

$0.1 \quad 0.2$

$0.1 \quad 0.2$

0.1

$\begin{array}{llll}0.000 & 0.7 & 1.3 & 1.26 \\ 0.000 & 0.7 & 1.4 & 1.29 \\ 0.005 & 0.2 & 1.4 & 1.21 \\ 0.000 & 0.6 & 1.6 & 1.29\end{array}$

$\begin{array}{llll}0.000 & 0.6 & 1.0 & 1.29\end{array}$

$\begin{array}{llll}0.000 & 0.5 & 1.2 & 1.32\end{array}$

$\begin{array}{llll}0.015 & 0.1 & 1.1 & 1.21\end{array}$

$\begin{array}{llll}0.000 & 0.4 & 1.3 & 1.32\end{array}$

$\begin{array}{llll}0.000 & 0.2 & 0.4 & 1.43\end{array}$

$\begin{array}{llll}0.000 & 0.2 & 0.5 & 1.57\end{array}$

$\begin{array}{llll}0.057 & 0.0 & 0.5 & 1.43\end{array}$

$\begin{array}{llll}0.053 & 0.0 & 0.5 & 1.43\end{array}$

0.3

0.4

0.5

0.2

0.5

0.3

0.3

0.3

0.3

0.3

0.4

0.1

0.3

0.8

0.6

0.9

0.9

0.4

0.3

0.5

0.5

0.001

0.008

0.485

0.063

0.2
0.1

0.7

1.14

1.14

1.05

1.14

$\begin{array}{llll}0.000 & 0.1 & 0.5 & 1.21\end{array}$

$\begin{array}{llll}0.003 & 0.1 & 0.6 & 1.29\end{array}$

$\begin{array}{llll}0.131 & -0.1 & 0.6 & 1.21\end{array}$

$\begin{array}{llll}0.071 & 0.0 & 0.6 & 1.21\end{array}$

$\begin{array}{llll}0.002 & 0.1 & 0.5 & 1.27\end{array}$

$\begin{array}{llll}0.004 & 0.1 & 0.7 & 1.27\end{array}$

$\begin{array}{llll}0.468 & -0.2 & 0.5 & 1.09\end{array}$

$\begin{array}{llll}0.166 & -0.1 & 0.7 & 1.18\end{array}$

$\begin{array}{llll}0.000 & 0.5 & 1.0 & 1.31\end{array}$

$\begin{array}{llll}0.000 & 0.3 & 0.9 & 1.23\end{array}$

$\begin{array}{llll}0.000 & 0.4 & 1.4 & 1.35\end{array}$

$\begin{array}{llll}0.000 & 0.5 & 1.4 & 1.38\end{array}$

$\begin{array}{llll}0.000 & 0.2 & 0.6 & 1.27\end{array}$

$\begin{array}{llll}0.006 & 0.1 & 0.6 & 1.20\end{array}$

$\begin{array}{llll}0.014 & 0.1 & 0.8 & 1.27\end{array}$

$\begin{array}{llll}0.004 & 0.2 & 0.9 & 1.33\end{array}$

$\begin{array}{llll}0.018 & 0.0 & 0.1 & 2.00\end{array}$

$\begin{array}{llll}0.041 & 0.0 & 0.2 & 2.00\end{array}$

$\begin{array}{llll}0.134 & 0.0 & 0.2 & 2.00\end{array}$

$\begin{array}{llll}0.740 & -0.1 & 0.1 & 2.00\end{array}$ 
Table 3 (continued)

\begin{tabular}{|c|c|c|c|c|c|c|c|}
\hline Outcome name & Comparison vs referent & $\begin{array}{l}\text { Mean } \\
\text { spending } \\
\text { (referent) }\end{array}$ & $\begin{array}{l}\text { Mean spend- } \\
\text { ing (compari- } \\
\text { son) }\end{array}$ & $\begin{array}{l}\text { Absolute } \\
\text { spending dif- } \\
\text { ference }\end{array}$ & $p$ value & Lower $95 \% \mathrm{CL}$ & Upper $95 \%$ CL \\
\hline \multicolumn{8}{|c|}{ Annual medical care spending } \\
\hline Any lagged Poly-ACH & $1+$ yrs vs 0 yrs & US\$8645 & US\$9762 & US\$1116 & 0.000 & US\$677 & US\$1556 \\
\hline \multirow[t]{3}{*}{ Count of lagged Poly-ACH } & 1 yr vs 0 yrs & US\$8645 & US\$9808 & US\$1163 & 0.000 & US\$560 & US\$1766 \\
\hline & 2 yrs vs 0 yrs & US\$8645 & US\$9815 & US\$1169 & 0.006 & US\$327 & US\$2011 \\
\hline & 3 yrs vs 0 yrs & US\$8645 & US\$9610 & US\$964 & 0.029 & US\$98 & US\$1830 \\
\hline
\end{tabular}

Source: Medicare enrollment and claims data, calendar year 2006-2017

$A M S$ altered mental status, Poly-ACH Polypharmacy: Use of Multiple Anticholinergic Medications in Older Adults (measure), $C L$ confidence limit, yrs years, $E R$ emergency room

${ }^{\text {a }}$ Risk ratio $=$ comparison mean/reference mean

Supplementary Information The online version contains supplementary material available at https://doi.org/10.1007/s40266-021-00901-2.

Acknowledgements This study was funded by Astellas Pharma. Medical writing/editorial support was provided by Alexis Mickle from Broadstreet HEOR and funded by the study sponsor.

\section{Declarations}

Funding This study was funded by Astellas Pharma Global Development.

Conflicts of interest/competing interests AS, DW, and AL are employees of Astellas and may own Astellas stock. NLC received consulting fees from Astellas Pharma for the conduct of this study. AJE (Medicus Economics) received funding from Astellas Pharma to develop, conduct, and analyze the study. LH is an employee of the Pharmacy Quality Alliance, the developer of the measure used in this research.

Ethics approval Not applicable.

Consent to participate Not applicable.

Consent for publication Not applicable.

Availability of data and material Researchers may request access to anonymized participant level data, trial level data, and protocols from Astellas sponsored clinical trials at www.clinicalstudydatarequest.com. For the Astellas criteria on data sharing, see https://clinicalstudydatareq uest.com/Study-Sponsors/Study-Sponsors-Astellas.aspx. The data that support the findings of this study are available from the Centers for Medicare and Medicaid Services, but restrictions apply to the availability of these data, which were used under license for the current study, and thus are not publicly available. Data are however available from the authors upon reasonable request and with permission of the Centers for Medicare and Medicaid Services.

Code availability Not applicable.

Author contributions All authors contributed to the study conception and design. Data analyses were performed by AJE. All authors contributed to the writing of the manuscript. All authors read and approved the final manuscript.
Open Access This article is licensed under a Creative Commons Attribution-NonCommercial 4.0 International License, which permits any non-commercial use, sharing, adaptation, distribution and reproduction in any medium or format, as long as you give appropriate credit to the original author(s) and the source, provide a link to the Creative Commons licence, and indicate if changes were made. The images or other third party material in this article are included in the article's Creative Commons licence, unless indicated otherwise in a credit line to the material. If material is not included in the article's Creative Commons licence and your intended use is not permitted by statutory regulation or exceeds the permitted use, you will need to obtain permission directly from the copyright holder. To view a copy of this licence, visit http://creativecommons.org/licenses/by-nc/4.0/.

\section{References}

1. Abrams P, Andersson K-E, Apostolidis A, Birder L, Bliss D, Brubaker L, et al. 6th International Consultation on Incontinence. Recommendations of the International Scientific Committee: evaluation and treatment of urinary incontinence, pelvic organ prolapse and faecal incontinence. Neurourol Urodyn. 2018;37(7):2271-2.

2. Eapen RS, Radomski SB. Review of the epidemiology of overactive bladder. Res Rep Urol. 2016;8:71-6.

3. Reynolds WS, Fowke J, Dmochowski R. The burden of overactive bladder on US public health. Curr Bladder Dysfunct Rep. 2016;11(1):8-13.

4. Ganz ML, Smalarz AM, Krupski TL, Anger JT, Hu JC, WittrupJensen KU, et al. Economic costs of overactive bladder in the United States. Urology. 2010;75(3):526-32.e18.

5. Lightner Deborah J, Gomelsky A, Souter L, Vasavada SP. Diagnosis and treatment of overactive bladder (non-neurogenic) in adults: AUA/SUFU Guideline Amendment 2019. J Urol. 2019;202(3):558-63.

6. Ganz ML, Liu J, Zou KH, Bhagnani T, Luo X. Real-world characteristics of elderly patients with overactive bladder in the United States. Curr Med Res Opin. 2016;32(12):1997-2005.

7. Ruxton K, Woodman RJ, Mangoni AA. Drugs with anticholinergic effects and cognitive impairment, falls and all-cause mortality in older adults: a systematic review and meta-analysis. Br J Clin Pharmacol. 2015;80(2):209-20.

8. Araklitis G, Cardozo L. Safety issues associated with using medication to treat overactive bladder. Expert Opin Drug Saf. 2017;16(11):1273-80. 
9. Salahudeen MS, Duffull SB, Nishtala PS. Anticholinergic burden quantified by anticholinergic risk scales and adverse outcomes in older people: a systematic review. BMC Geriatr. 2015;15:31.

10. Landi F, Russo A, Liperoti R, Cesari M, Barillaro C, Pahor M, et al. Anticholinergic drugs and physical function among frail elderly population. Clin Pharmacol Ther. 2007;81(2):235-41.

11. Gray SL, Anderson ML, Dublin S, Hanlon JT, Hubbard R, Walker R, et al. Cumulative use of strong anticholinergics and incident dementia: a prospective cohort study. JAMA Intern Med. 2015;175(3):401-7.

12. Fox C, Richardson K, Maidment ID, Savva GM, Matthews FE, Smithard D, et al. Anticholinergic medication use and cognitive impairment in the older population: the Medical Research Council Cognitive Function and Ageing Study. J Am Geriatr Soc. 2011:59(8):1477-83.

13. The 2019 American Geriatrics Society Beers Criteria ${ }^{\circledR}$ Update Expert Panel. American Geriatrics Society 2019 Updated AGS Beers Criteria ${ }^{\circledR}$ for Potentially Inappropriate Medication Use in Older Adults. J Am Geriatr Soc. 2019;67(4):674-694. https://doi. org/10.1111/jgs.15767.

14. Lozano-Ortega G, Schermer CR, Walker DR, Szabo SM, Rogula B, Deighton AM, et al. Fall/fracture-related healthcare costs and their association with cumulative anticholinergic burden in people with overactive bladder. Pharmacoecon Open. 2021;5(1):45-55.

15. Campbell NL, Perkins AJ, Bradt P, Perk S, Wielage RC, Boustani MA, et al. Association of anticholinergic burden with cognitive impairment and health care utilization among a diverse ambulatory older adult population. Pharmacotherapy. 2016;36(11):1123-31.

16. Pharmacy Quality Alliance. Polypharmacy: use of multiple anticholinergic medications in older adults (POLY-ACH) 2018. https://www.pqaalliance.org/measures-overview\#poly-ach. Accessed 14 Oct 2021.

17. (CMS) CfMMS. Center for Medicare \& Medicaid Services (CMS) Medicare Enrollment Dashboard 2019. https://www.cms. gov/Research-Statistics-Data-and-Systems/Statistics-Trends-andReports/Dashboard/Medicare-Enrollment/Enrollment\%20Das hboard.html. Accessed 14 Oct 2021.

18. Richardson K, Fox C, Maidment I, Steel N, Loke YK, Arthur A, et al. Anticholinergic drugs and risk of dementia: case-control study. BMJ. 2018;361:k1315.

19. Szabo SM, Gooch K, Schermer C, Walker D, Lozano-Ortega $\mathrm{G}$, Rogula B, et al. Association between cumulative anticholinergic burden and falls and fractures in patients with overactive bladder: US-based retrospective cohort study. BMJ Open. 2019;9(5):e026391.

20. Ng DB, Espinosa R, Johnson SJ, Walker D, Gooch K. The impact of persistence with mirabegron usage vs switching to onabotulinumtoxinA on healthcare costs and resource utilization in patients with overactive bladder in the United States. J Med Econ. 2017;20(12):1272-80.

21. Lin C-S, Khan H, Chang R-Y, Liao W-C, Chen Y-H, Siao S-Y, et al. A study on the impact of poor medication adherence on health status and medical expense for diabetes mellitus patients in Taiwan: a longitudinal panel data analysis. Medicine. 2020;99(26):e20800-e.

22. Dunn A, Grosse SD, Zuvekas SH. Adjusting health expenditures for inflation: a review of measures for health services research in the United States. Health Serv Res. 2018;53(1):175-96.

23. Manning WG, Mullahy J. Estimating log models: to transform or not to transform? J Health Econ. 2001;20(4):461-94.

24. Elixhauser A, Steiner C, Harris DR, Coffey RM. Comorbidity measures for use with administrative data. Med Care. 1998;36(1):8-27.

25. Coupland CAC, Hill T, Dening T, Morriss R, Moore M, Hippisley-Cox J. Anticholinergic drug exposure and the risk of dementia: a nested case-control study. JAMA Intern Med. 2019;179(8):1084-93.

26. Suehs BT, Caplan EO, Hayden J, Ng DB, Gaddy RR. The relationship between anticholinergic exposure and falls, fractures, and mortality in patients with overactive bladder. Drugs Aging. 2019;36(10):957-67.

27. Cisternas MG, Foreman AJ, Marshall TS, Runken MC, Kobashi $\mathrm{KC}$, Seifeldin R. Estimating the prevalence and economic burden of overactive bladder among Medicare beneficiaries prior to Medicare Part D coverage. Curr Med Res Opin. 2009;25(4):911-9.

28. Centers for Medicare \& Medicaid Services. CMS fast facts: CIMS Program data: populations 2021. https://www.cms.gov/researchstatistics-data-systems/cms-fast-facts/cms-fast-facts-mobile-site. Accessed 14 Oct 2021.

29. Medicare Payment Advisory Commission. Report to Congress: Medicare payment policy 2020. http://medpac.gov/docs/defaultsource/reports/mar21_medpac_report_to_the_congress_sec.pdf. Accessed 14 Oct 2021. 